Unveiling the optical properties of a metamaterial synthesized by electron-beam-induced deposition

This content has been downloaded from IOPscience. Please scroll down to see the full text. 2016 Nanotechnology 27025705

(http://iopscience.iop.org/0957-4484/27/2/025705)

View the table of contents for this issue, or go to the journal homepage for more

Download details:

IP Address: 160.45.66.177

This content was downloaded on 11/02/2016 at 10:18

Please note that terms and conditions apply. 


\title{
Unveiling the optical properties of a metamaterial synthesized by electron-beam- induced deposition
}

\author{
P Woźniak ${ }^{1,2,4}$, K Höflich ${ }^{1,3,4}$, G Brönstrup ${ }^{1,3}$, P Banzer ${ }^{1,2}$, \\ S Christiansen ${ }^{1,3}$ and $\mathbf{G}$ Leuchs ${ }^{1,2}$
}

\footnotetext{
${ }^{1}$ Max Planck Institute for the Science of Light, Günther-Scharowsky-Str.1, D-91058 Erlangen, Germany

${ }^{2}$ Institute of Optics, Information and Photonics, Friedrich-Alexander-University Erlangen-Nuremberg,

Staudtstr. 7/B2, D-91058 Erlangen, Germany

${ }^{3}$ Institute of Nanoarchitectures for Energy Conversion, Helmholtz Centre Berlin for Materials and Energy, Hahn-Meitner-Platz 1, D-14109 Berlin, Germany
}

E-mail: pawel.wozniak@mpl.mpg.de

Received 21 September 2015

Accepted for publication 26 October 2015

Published 2 December 2015

\begin{abstract}
Direct writing using a focused electron beam allows for fabricating truly three-dimensional structures of sub-wavelength dimensions in the visible spectral regime. The resulting sophisticated geometries are perfectly suited for studying light-matter interaction at the nanoscale. Their overall optical response will strongly depend not only on geometry but also on the optical properties of the deposited material. In the case of the typically used metal-organic precursors, the deposits show a substructure of metallic nanocrystals embedded in a carbonaceous matrix. Since gold-containing precursor media are especially interesting for optical applications, we experimentally determine the effective permittivity of such an effective material. Our experiment is based on spectroscopic measurements of planar deposits. The retrieved permittivity shows a systematic dependence on the gold particle density and cannot be sufficiently described using the common Maxwell-Garnett approach for effective medium.
\end{abstract}

S Online supplementary data available from stacks.iop.org/nano/27/025705/mmedia

Keywords: electron beam induced deposition, metamaterials, nanocomposite

(Some figures may appear in colour only in the online journal)

\section{Introduction}

Nowadays, the fast development of nanofabrication methods provides access to functional structures with geometries of sub-wavelength dimensions even in the visible regime. Thereby, fundamental questions of light-matter interaction

${ }^{4}$ P Woźniak and K Höflich contributed equally to this work. can be addressed as done within the intensely studied field of nano-optics. In particular, nanostructures made of materials having a free electron gas [1], excitable to collective oscillations by light (plasmon-polaritons), provide the possibility of tailored light manipulation and concentration [2-4]. Such sub-wavelength structures are key for the design of metamaterials [5]. However, the fabrication of truly three-dimensional nanostructures still represents a significant challenge. While, for example, direct laser writing provides threedimensional and purely metallic [6] as well as dielectric [7] structures, it is limited primarily not in terms of optical resolution, but most likely due to diffusion constraints during the electroless plating. In contrast, direct writing using a 
focused electron beam is capable of delivering three-dimensional features, smaller than tens of nanometer in a highly flexible and precise single-step process [8-10]. The electron beam induced deposition (EBID) process is based on local decomposition of inserted precursor-gas molecules by an electron beam within a vacuum chamber of a scanning electron microscope. Since typical precursors are of the metalorganic type, not only their metallic constituents remain after deposition, although this would be the ideal case. Instead, a substantial amount of ligand components as well as elements of the residual gas in the vacuum chamber (carbon, oxygen and hydrogen) are incorporated $[8,11]$. Thus, the resulting composite can be viewed as a metamaterial or an effective material by itself, consisting of single-crystalline nanoparticles (e.g. gold) embedded in a carbonaceous matrix [10]. While the electric properties of granular materials such as the EBID material are well understood [12] and proven to be promising for sensing applications [13, 14], a complete optical description of EBID metamaterials based on a combined experimental and theoretical study has not been reported yet. This is caused by the relatively small areas which can be conventionally fabricated with the EBID process, not allowing for optical characterization with standard methods such as ellipsometry. First attempts using microellipsometry showed a behaviour close to a Maxwell-Garnett effective medium for a gold-containing EBID material [15]. Recently, the Maxwell-Garnett theory was also used to describe material properties of helices made of platinumEBID composite [16] to numerically study their circular dichroism. While the overall agreement between experiment and numerical simulations was reasonable, distinct deviations became obvious for both the long wavelength range and the actual strength of the observed optical activity [16]. This trend was also emphasized in a numerical study of similar composite materials [17].

Here, we present the results of spectrometric measurements in combination with a numerical algorithm based on the transfer-matrix approach for retrieval of the permittivity of gold-EBID $10 \times 10 \mu \mathrm{m}^{2}$ pad-like deposits (see figure 1(c)) . The investigation concerns deposits of different thicknesses to systematically study the dependence of the permittivity on the density of the nanoinclusions in the carbonaceous matrix. In addition, the comparison of analytical results based on Mie theory with an effective medium model such as MaxwellGarnett proves that although the dipole resonance of the gold particles dominantly contributes to the optical response of the EBID composite, the conventional Maxwell-Garnett approach cannot be employed to describe the effective optical properties of the composite. The presented experimentally retrieved permittivity is expected to give better understanding of optical performance of nanostructures fabricated via EBID, paving the way for sophisticated nano-optic applications.

\section{Sample preparation}

The experimental investigation started with the fabrication of a substrate which consists of a $170 \mu \mathrm{m}$ thick glass (BK7) plate

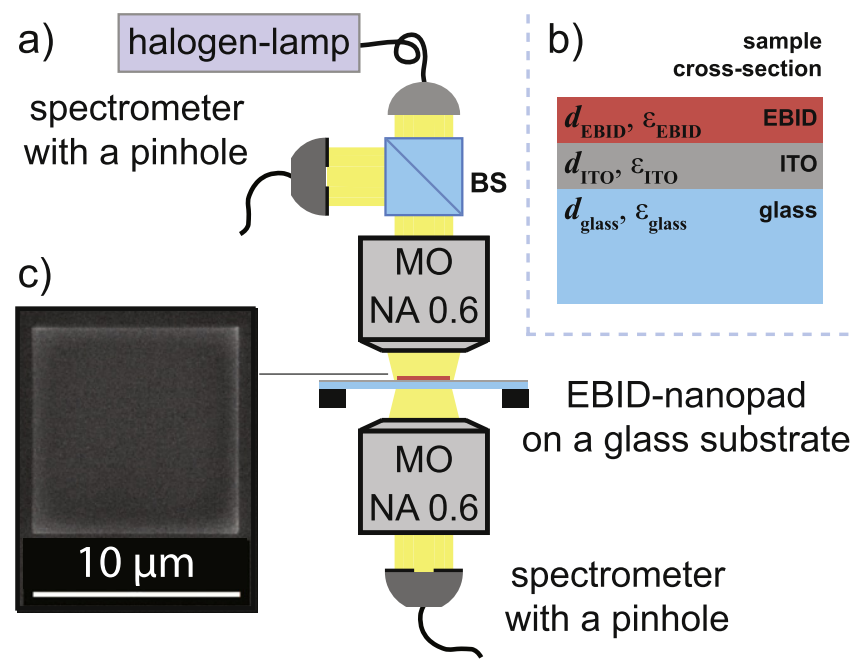

Figure 1. (a) Microscopy setup for spectral measurements of EBID nanopads. The sample is illuminated with white light emitted by a halogen-lamp. A beam splitter (BS) between the lamp and the first microscope objective (MO) guides part of the reflected light onto a detector. The central part of the nanopad is imaged onto a pinhole with a diameter of $600 \mu \mathrm{m}$ placed in front of the detectors. (b) Crosssection through the nanopad and the substrate (layer thicknesses are not drawn to scale). (c) Scanning electron micrograph of an investigated EBID pad.

with a conductive thin layer of indium tin oxide (ITO) on top ${ }^{5}$. Both materials were characterized using ellipsometry and show typical properties ${ }^{6}$. The same ellipsometry measurement was used to determine most accurately the thickness of the ITO coating $(36 \mathrm{~nm})$.

The EBID process itself was carried out in a vacuum chamber of a dual-beam instrument (FEI Strata DB 235) equipped with a gas-injection system for dimethyl-gold(III)acetyl-acetonate $\left(\mathrm{Me}_{2} \mathrm{Au}(\mathrm{acac})\right)$ as the precursor gas [18]. The injected precursor molecules adsorb, diffuse and desorb onto the sample surface where they are locally cracked by the focused electron beam [19]. Thereby, the EBID technique not only allows for the realization of complex three-dimensional geometries $[16,20]$ but also for depositing them on even nonplanar substrates, if these are at least weakly conductive [10]. During the molecule dissociation, their non-volatile parts form the deposit while the volatile constituents are pumped out [18].

The deposition process depends on the energy and current of the electron-beam, and on the dwell time (the time the beam resides at each position) as well as on the scanning raster. All these factors were previously optimized for fabrication of high-resolution three-dimensional nanostructures [10]. The aim in the current work was to keep the deposition parameters of the pad deposits as close as possible near the range used for nanostructures to unveil their material properties. Correspondingly, the retrieved permittivity reliably describes the material of nanostructures written by EBID.

\footnotetext{
5 Commercial ITO-on-glass substrate was avoided due to undefined thickness of the ITO coating and its pronounced crystallinity causing strong scattering effects.

6 see supporting information available at stacks.iop.org/nano/27/025705/ mmedia.
} 
Since the optimized parameters would lead to an unrealistically long deposition time for such deposits, the dwell time was drastically reduced down to $1 \mu$ s and the lateral sizes were restricted to $10 \times 10 \mu \mathrm{m}^{2}$ while maintaining the other parameters (see scanning electron micrograph of a typical EBID pad in figure 1(c)). For the systematic investigation, EBID deposits with thicknesses ranging from $15 \mathrm{~nm}$ up to $50 \mathrm{~nm}$ (far below the Fabry-Pérot cavity condition) were fabricated and topographically characterized by atomic force microscopy (AFM). The AFM scans confirmed that at the edges all pads have increased thickness with respect to the perfectly smooth and flat interior. This is caused by the low pressure of the precursor gas [18], resulting in a mass-transport-limited (also called molecule-limited) deposition regime [21-23]. In this regime all available precursor molecules within the square deposition region are consumed and dissociated by the primary and the secondary electrons. This leads to a decreased deposition rate in the central area as well as to stronger codeposition of carbon from the residual chamber gases $[19,24]$. The diffusion of the intact precursor molecules outside the irradiated region causes an increased precursor supply at the edges, leading to enhanced deposition there [22]. Hence, each fabricated pad exhibits a $9 \times 9 \mu \mathrm{m}^{2}$ large surface area of high quality and constant thickness. These were utilized for spectroscopic measurements in an optical microscopic setup.

\section{Methods}

To measure optical spectra, a home-built microscopic setup was used (see figure 1(a)). A halogen lamp emitted unpolarized and noncoherent light within the spectral range of interest between $480 \mathrm{~nm}$ and $900 \mathrm{~nm}$. The sample was illuminated using a $60 \times$ microscope objective, which also collected the reflected light. For separation of the incident and the reflected light, a beam splitter was used between the source and the objective. The position of the microscope objective relative to the sample surface was carefully adjusted to obtain plane-wave illumination at normal incidence and to image the sample surface onto the detector plane. The area of interest could be selected using a pinhole in the image plane. The size of this additional aperture was chosen to be $600 \mu \mathrm{m}$ in diameter, guaranteeing the measurement of the signal solely from the flat inner part of the deposit, while maintaining a sufficient signal-to-noise ratio. Each EBID pad was measured separately by placing it on the optical axis such that the propogation of the incoming plane-wave was normal with regard to the sample surface. In the same way, the light transmitted through the sample was measured.

The experimental scheme described above permits interpreting the measurement data as spectra of reflected $\left(I_{\mathrm{R}}\right)$ and transmitted $\left(I_{\mathrm{T}}\right)$ intensities of an incoming plane-wave $\left(I_{0}\right)$ by a multilayer system (see figure 1(b)). Such a structure can be analytically described using a transfer matrix (see footnote 6):

$$
\left[\begin{array}{c}
I_{\mathrm{R}} \\
I_{\mathrm{T}}
\end{array}\right]=\left[\begin{array}{c}
R_{\mathrm{EBID}} R_{\mathrm{ITO}} R_{\text {glass }} \\
T_{\mathrm{EBID}} T_{\mathrm{ITO}} T_{\text {glass }}
\end{array}\right] I_{0},
$$

where $R$ and $T$ are reflection and transmission matrices describing propagation and multiple reflection of light within the EBID layer, the ITO layer and the glass substrate, respectively. Each matrix contains information about the geometrical (thickness $d$ ) and optical (permittivity $\epsilon$ ) properties of the corresponding layer. The only unknowns in the equation above are the real and imaginary part of $\epsilon_{\mathrm{EBID}}$. All further parameters describing the ITO-on-glass substrate as well as the EBID layer thicknesses are determined experimentally. Consequently, the optical properties of the EBID material can be retrieved from measurements of reflection and transmission. Due to the sub-wavelength thickness, the light within the EBID and the ITO layers $\left(R_{\mathrm{EBID}}, R_{\mathrm{ITO}}, T_{\mathrm{EBID}}\right.$ and $\left.T_{\mathrm{ITO}}\right)$ must be described as coherent [25] in the investigated spectral range. In contrast, the glass substrate is two orders of magnitude thicker than $\lambda$. Therefore, and due to the used light source, light propagation across the entire sample requires a semi-coherent description [26]. Furthermore, according to equation (1) the measured values $I_{\mathrm{R}}$ and $I_{\mathrm{T}}$ have to be normalized with respect to the incident intensity $I_{\mathrm{I}}$. To this end, the reflection from and the transmission through the ITO-on-glass substrate was measured and the theoretical reflectance and transmittance coefficients were calculated (see footnote 6).

Though the calculation of the sample's reflectance and transmittance is straightforward if the complex permittivity of the EBID layer is known, the inverse problem is not. In addition, there are several distinct variations of $\epsilon_{\mathrm{EBID}}$ which result in the same reflectance and transmittance of the sample. Therefore, this inverse problem was tackled using a brute force search, i.e., calculating the reflectance and transmittance of the sample for several thousand possible complex values of $\epsilon_{\mathrm{EBID}}$ for each wavelength and comparing them with the experimental results. Thereby, all local minima in the twodimensional permittivity-space corresponding to the respective smallest deviation from the measured spectra were identified. By subsequent refining of the region around the minima, several steady solutions of $\epsilon_{\mathrm{EBID}}(\lambda)$ were found. However, there is only one physical solution which has to be determined. Accordingly, all dispersions which exhibit a constant increase of the real part of the refractive index ( $\mathrm{dRe}[n] / \mathrm{d} \lambda=$ const where $n=\sqrt{\epsilon})$ in the whole investigated spectral range are excluded. Such solutions have unrealistically high values and appear repeatedly while extending the plane of possible solutions (see footnote 6). In contrast, the only dispersion which does not follow this tendency has values in a reasonable range and meaningfully reflects the inner structure of the EBID composite. 

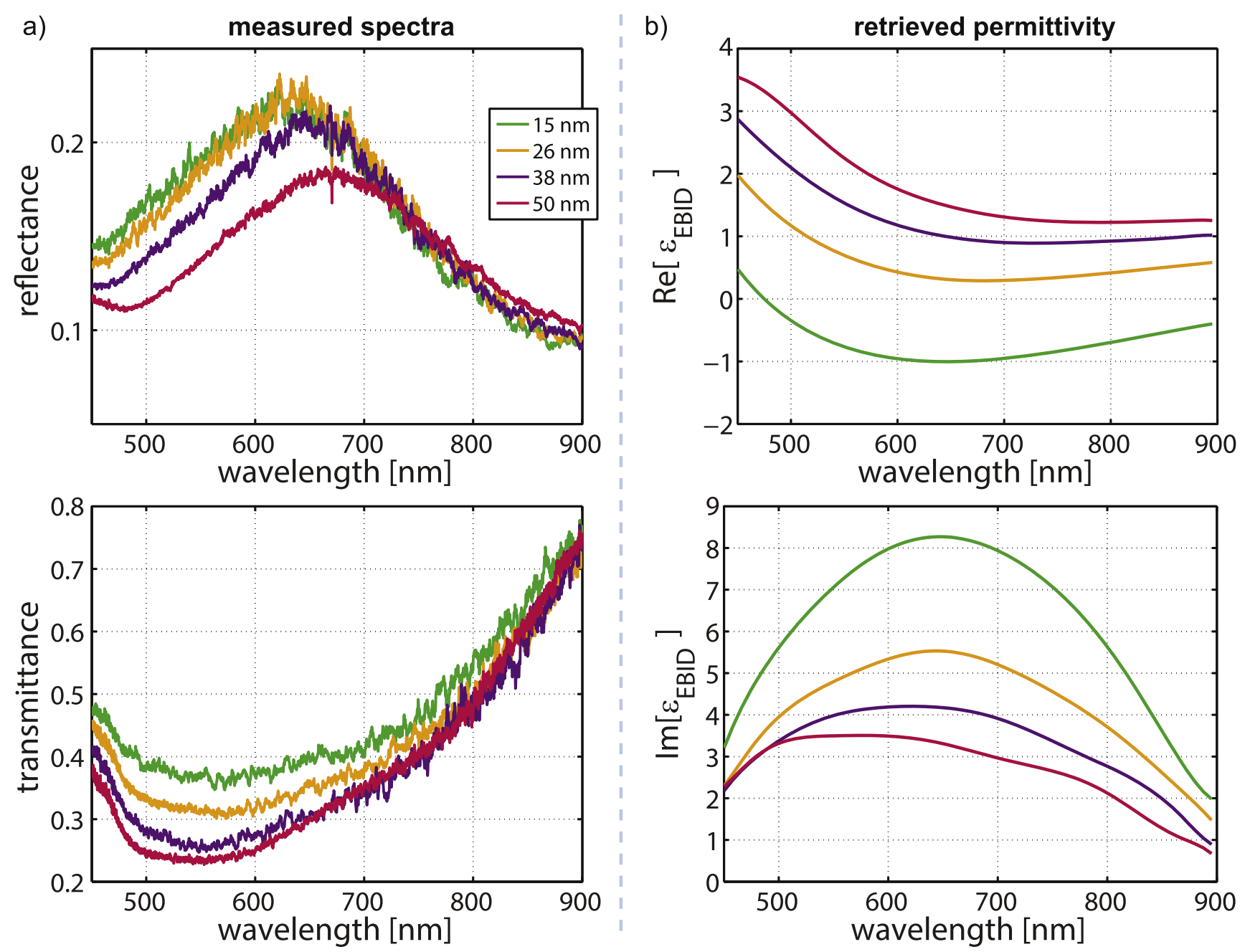

Figure 2. (a) Experimental reflectance and transmittance spectra of EBID nanopads of thicknesses between $15 \mathrm{~nm}$ and $50 \mathrm{~nm}$. (b) Retrieved permittivity as a function of the pad thickness. For the sake of clarity, the measurement noise is removed by fitting the retrieved real and imaginary parts of $\epsilon_{\mathrm{EBID}}(\lambda)$ with a polynomial function. The green curve of the thinnest deposit is expected to approximate the best material properties of nanostructures, fabricated with EBID.

\section{Results and discussion}

Figure 2(a) shows the experimental reflectance and transmittance data of the EBID-ITO-glass system for different deposit thicknesses. All reflectance spectra exhibit distributions with a maximum value observed at around $620 \mathrm{~nm}$, which slightly red-shifts for increasing layer thickness. Likewise, the transmittance spectra feature minima at around $550 \mathrm{~nm}$ and also show the expected decrease in transmittance for thicker pads. The corresponding real and imaginary parts of the retrieved permittivity are presented in figure 2(b) (for the sake of clarity, the measurement noise is removed by fitting the retrieved real and imaginary parts of $\epsilon_{\mathrm{EBID}}(\lambda)$ with polynomial functions) and show a clear systematic dependence on the thickness of the EBID pads. The real part of $\epsilon_{\mathrm{EBID}}$ decreases when decreasing deposit thickness, approaching even metallic behaviour for the thinnest pad. In contrast, the imaginary part increases with decreasing thickness and moves to form a broad peak centred at around
$650 \mathrm{~nm}$. This tendency implies that the EBID composite behaves more and more dielectrically for thicker deposits.

This observation is consistent with a chemisorption of the gold-containing precursor molecules onto the sample surface together with possibly decreasing vapour pressure over the long deposition times [19, 24]. As mentioned, the concave shape of the pads implies a mass-transport-limited deposition regime [22] in which excess electrons start to dissociate residual gases-mainly hydrocarbons present in the vacuum chamber. For typical vacuum pressures around $10^{-6} \mathrm{hPa}$, still a large number of molecules sufficient to form one monolayer per second impinges onto the substrate only from the residual gases [19] and possibly due to a longer resident time of some precursor-gas ligands at the irradiated spot [21]. Thereby, after the depletion of the chemisorbed initial layer, the codeposition of carbon, oxygen and hydrogen becomes dominant and increases the relative carbon content in the thicker pads. For a long deposition time, this effect becomes particularly important if the precursor vapour pressure decreases with time. Thus, the carbon content of the deposits 


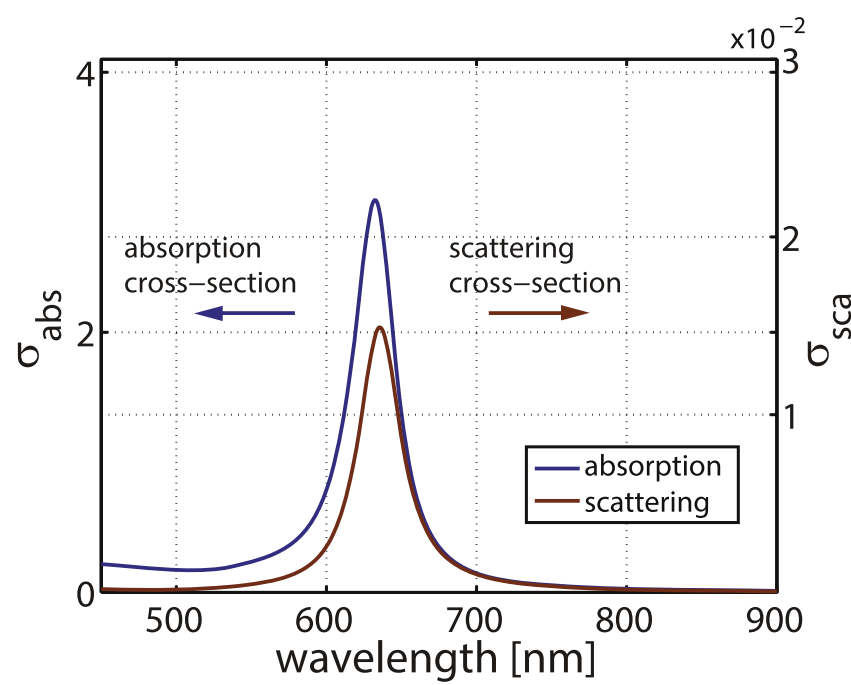

Figure 3. Mie scattering and absorption cross-sections of isolated gold particles embedded in diamond environment under plane-wave excitation. The particles have a mean diameter of $4 \mathrm{~nm}$ with a lognormal size distribution of $50 \%$ standard deviation.

rises along with the deposition time and with the deposit thickness [24]. As a consequence, properties of the EBID composite can be tuned by the density of the gold inclusions, from strongly dielectric to slightly metallic optical behaviour.

In that respect, the permittivity of the thinnest pad is expected to correspond to the material properties of nanostructures which can be fabricated using EBID. Fine structures (e.g. pillars or needles) are fabricated optimally with a dwell time even up to $720 \mu$ s, initiating local heating, while staying in the mass-transport-limited deposition regime. Furthermore, the slender geometry, together with the overall short deposition time, supports the heating effect, which ensures a relatively high content of gold around 28 at $\%$ [10].

Concerning the spectral characteristic of the retrieved permittivity, the prominent resonance in $\operatorname{Im}\left[\epsilon_{\mathrm{EBID}}\right]$ at around $650 \mathrm{~nm}$ can be attributed to the dipole resonance of the gold nanoparticles within the carbonaceous matrix. The mean particle diameter of the singly crystalline particles varies around $4 \mathrm{~nm}$ ranging approximately from $2 \mathrm{~nm}$ to $7-8 \mathrm{~nm}$ with a decreasing number of larger particles [10, 27]. Correspondingly, figure 3 shows Mie cross-sections of absorption (blue line) and of scattering (brown line) for isolated spherical gold particles of a mean diameter of $4 \mathrm{~nm}$ with a lognormal size distribution of $50 \%$ standard deviation under plane-wave illumination (see footnote 6). The EBID matrix is approximated by non-absorbing diamond [28] (solid line) while gold properties are taken from [29]. As expected, the Mie crosssections are dominated by an absorptive dipole resonance around $630 \mathrm{~nm}$, which corresponds to the absorption properties retrieved for the EBID matrix. In this size regime the shift of the resonance position due to the nanoparticle size distribution is negligible. Thus, the Mie resonance is comparatively narrow, indicating that the broadening of the metamaterial resonance is due to further loss mechanisms besides the effect of the size distribution and the intrinsic absorption of the gold inclusions. In that respect, absorption of

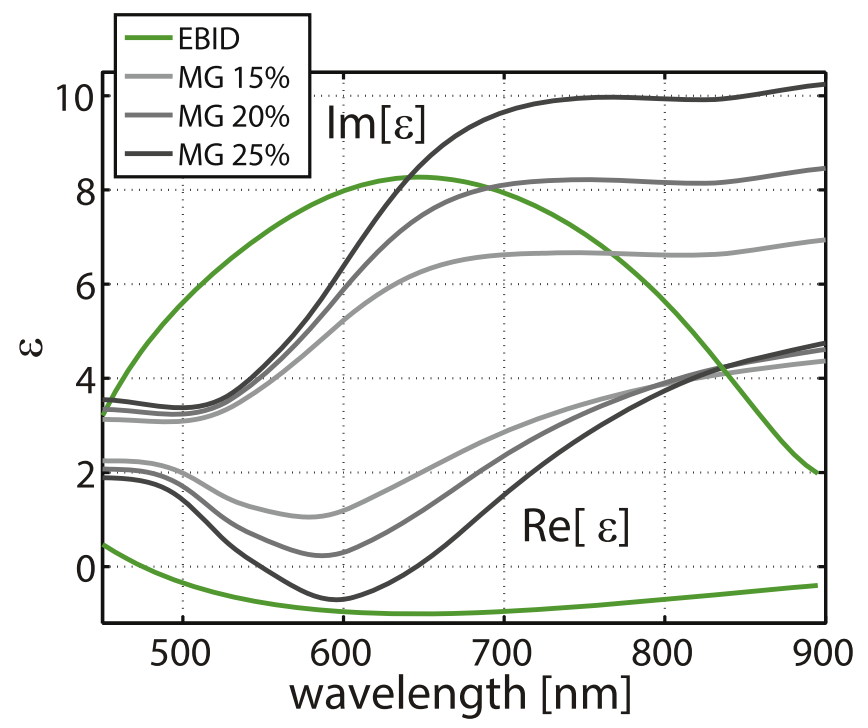

Figure 4. Comparison of the retrieved permittivity of the EBID material (green) with the Maxwell-Garnett effective medium theory for three different volume filling fractions of $15 \%, 20 \%$ and $25 \%$.

the carbonaceous matrix as well as interaction between the particles, which are not included in the classical Mie approach, play a significant role. Careful analytical considerations [30] show that tiny losses of the matrix will not only slightly red-shift the peak position but also cause strong broadening of the resonance. Therefore, losses of the carbonaceous matrix, especially for the thicker deposits, are responsible for the very broad resonance of in $\operatorname{Im}\left[\epsilon_{\mathrm{EBID}}\right]$. Based on these results, description of $\epsilon_{\mathrm{EBID}}(\lambda)$ using an effective medium approach based on dipoles in a dielectric environment seems to be promising. Considering the inner structure of the EBID material (randomly distributed metallic nanocrystals of deep sub-wavelength dimensions within a dielectric matrix), the Maxwell-Garnett theory [31] constitutes the obvious choice. For that purpose the matrix material is described as amorphous carbon [32] to account for the matrix loss, and gold properties are taken again from [29]. Since the actual value of the volume filling factor of gold in the composite is not known, it can therefore act as tuning factor. Figure 4 shows the comparison between the retrieved permittivity of the thinnest pad and the Maxwell-Garnett effective media for three different values of filling factor: $15 \%, 20 \%, 25 \%$. None of the possible combinations can reproduce the retrieved $\epsilon_{\mathrm{EBID}}(\lambda)$. Neither tuning of the filling factor nor substitution of the material properties of gold and the carbonaceous matrix (including absorption) from other material databases significantly changes the output of the Maxwell-Garnett calculations. This indicates that the EBID composite does not meet the Maxwell-Garnett conditions $[17,31]$. Indeed, the particles can be safely described within the quasistatic approximation; the absorptive dipole-type resonance fits well to the Maxwell-Garnett approach considering pure dipole-dipole interaction under the assumption of negligible scattering. However, well separation of the particles, required by the Maxwell-Garnett cannot be taken for granted. The close spacing between the gold inclusions 
will presumably cause both, not only near-field interactions [17], but also local electron tunnelling through the carbonaceous matrix. As proven for the electrical applications of EBID materials, the gold nanocrystals are not electrically isolated within the carbonaceous matrix, but instead, electron transport, due to thermally assisted tunneling of electrons, takes place [13]. Furthermore, the mass-limited deposition regime and hence the varying amount of co-deposited carbon influences the local particle density. An appropriate choice for the extension of Maxwell-Garnett seems to be the introduction of self-consistency, and thereby the iterative correction of the properties of the carbonaceous matrix by the effective permittivity itself [33]. In addition, the formation effect of differently shaped particle aggregates and their influence of higher order multipolar contributions need to be accounted for as well [17, 34]. However, considering all these effects together and due to the lack of knowledge concerning the optical properties of the carbonaceous matrix, a consistent effective material approach relying on only few and physically meaningful parameters still represents a significant challenge.

\section{Conclusions}

In summary, the dielectric function of an EBID material consisting of single-crystalline gold particles dispersed in a carbonaceous matrix is studied experimentally. The retrieved effective permittivities show a systematic dependence on the layer thickness and, thus, the particle density within the deposit. The effective permittivity for the highest metal content represents the best approximation of the material properties of EBID nanostructures. While the prominent absorption feature present in the retrieved imaginary parts of the EBID permittivities could be attributed to the dipole resonances of the embedded gold nanoparticles via Mie calculations, the optical properties of the material cannot be described by using a standard Maxwell-Garnett approach. Remarkably, this material also shows a very interesting optical behaviour at wavelengths around $450 \mathrm{~nm}$ where the real part of its permittivity exhibits a zero-crossing, which might require more attention. While the presented study is based on $\mathrm{Me}_{2} \mathrm{Au}$ (acac) as an exemplary gas precursor, the discussed method can be applied to any type of EBID-based metamaterial fabricated using metal-organic precursors. Thereby, the numerical and experimental study of complex nanostructures made of EBID metamaterials can be envisaged.

\section{Acknowledgments}

The research leading to these results received funding from the Helmholtz Association within the Helmholtz Postdoc Programme as well as from the European Union Seventh Framework Programme under Grant Agreement No. 280566 (www.univsem.eu) and under Grant Agreement No. 258868 (www.lcaos.eu).

\section{References}

[1] Boltasseva A and Atwater H A 2011 Low-loss plasmonic metamaterials Science 331 290-1

[2] Banzer P, Peschel U, Quabis S and Leuchs G 2010 On the experimental investigation of the electric and magnetic response of a single nano-structure Opt. Express 18 10905-23

[3] Schuller J A, Barnard E S, Cai W, Jun Y C, White J S and Brongersma M L 2010 Plasmonics for extreme light concentration and manipulation Nat. Mater. 9 193-204

[4] Baffou G and Quidant R 2014 Nanoplasmonics for chemistry Chem. Soc. Rev. 43 3898-907

[5] Cai W and Shalaev V 2010 Optical Metamaterials (New York: Springer)

[6] Gansel J K, Thiel M, Rill M S, Decker M, Bade K, Saile V, von Freymann G, Linden S and Wegener M 2009 Gold helix photonic metamaterial as broadband circular polarizer Science 325 1513-5

[7] Thiel M, Fischer H, von Freymann G and Wegener M 2010 Three-dimensional chiral photonic superlattices. Opt. Lett. 35 166-8

[8] Utke I, Moshkalev S and Russell P 2012 Nanofabrication Using Focused Ion and Electron Beams: Principles and Applications (Oxford: Oxford University Press)

[9] Huth M, Porrati F, Schwalb Ch, Winhold M, Sachser R, Dukic M, Adams J and Fantner G 2012 Focused electron beam induced deposition: A perspective Beilstein J. Nanotechnology 3 597-619

[10] Höflich K, Yang R B, Berger A, Leuchs G and Christiansen S 2011 The direct writing of plasmonic gold nanostructures by electron-beam-induced deposition. Adv. Mater. 23 2657-61

[11] Botman A, Mulders J J L and Hagen C W 2009 Creating pure nanostructures from electron-beam-induced deposition using purification techniques: a technology perspective Nanotechnology 20372001

[12] Beloborodov I, Lopatin A, Vinokur V and Efetov K 2007 Granular electronic systems Rev. Mod. Phys. 79 469-518

[13] Huth M, Kolb F and Plank H 2014 Dielectric sensing by charging energy modulation in a nano-granular metal Appl. Phys. A 117 1689-96

[14] Kolb F, Schmoltner K, Huth M, Hohenau A, Krenn J, Klug A, List E J W and Plank H 2013 Variable tunneling barriers in FEBID based PtC metal-matrix nanocomposites as a transducing element for humidity sensing Nanotechnology 24305501

[15] Utke I, Jenke M G, Röling Ch, Thiesen P H, Iakovlev V, Sirbu A, Mereuta A, Caliman A and Kapon E 2011 Polarisation stabilisation of vertical cavity surface emitting lasers by minimally invasive focused electron beam triggered chemistry Nanoscale 3 2718-22

[16] Esposito M, Tasco V and Cuscuna M 2014 Nanoscale 3D chiral plasmonic helices with circular dichroism at visible frequencies ACS Photonics 2 105-14

[17] Etrich Ch, Fahr S, Hedayati M K, Faupel F, Elbahri M and Rockstuhl C 2014 Effective optical properties of plasmonic nanocomposites Materials 7 727-41

[18] Wnuk J D, Gorham J M, Rosenberg S G, van Dorp W F, Madey T E, Hagen C W and Fairbrother D H 2010 Electron beam irradiation of dimethyl-(acetylacetonate) gold(III) adsorbed onto solid substrates J. Appl. Phys. 107054301

[19] Utke I and Gölzhäuser A 2010 Klein, minimal-invasiv, direkt: Elektronen induzieren lokale Reaktionen adsorbierter funktioneller Moleküle auf der Nanometerskala Angew. Chem. 122 9516-8

[20] Höflich K, Becker M, Leuchs G and Christiansen S 2012 Plasmonic dimer antennas for surface enhanced Raman scattering Nanotechnology 23185303 
[21] Szkudlarek A, Szmyt W, Kapusta C and Utke I 2014 Lateral resolution in focused electron beam-induced deposition: Scaling laws for pulsed and static exposure Appl. Phys. A 117 1715-26

[22] Winkler R, Fowlkes J, Szkudlarek A, Rack P D and Plank H 2014 The nanoscale implications of a molecular gas beam during electron beam induced deposition ACS Appl. Mater. Interfaces 6 2987-95

[23] Plank H, Haber T, Gspan Ch, Kothleitner G and Hofer F 2013 Chemical tuning of PtC nanostructures fabricated via focused electron beam induced deposition Nanotechnology 24175305

[24] Bernau L, Gabureac M, Erni R and Utke I 2010 Tunable nanosynthesis of composite materials by electron-impact reaction Angew. Chem. Int. Ed. 49 8880-4

[25] Moharam M G, Pommet D A, Grann E B and Gaylord T K 1995 Stable implementation of the rigorous coupled-wave analysis for surface-relief gratings: enhanced transmittance matrix approach J. Opt. Soc. Am. A 12 1077-86

[26] Harbecke B 1986 Coherent and incoherent reflection and transmission of multilayer structures Appl. Phys. B 39 $165-70$
[27] Riazanova A V, Rikers Y G M, Mulders J J L and Belova L M 2012 Pattern shape control for heat treatment purification of electron-beam-induced deposition of gold from the $\mathrm{Me} 2 \mathrm{Au}$ (acac) precursor Langmuir 28 6185-91

[28] Peter F 1923 Über Brechungsindizes und Absorptionskonstanten des Diamanten zwischen 644 und $226 \mathrm{~m} \mu$ Z. Phys. 15 358-68

[29] Johnson P B and Christy R W 1972 Optical constants of the noble metals Phys. Rev. B 6 4370-9

[30] Quinten M and Rostalski J 1996 Lorenz-Mie Theory for Spheres Immersed in an absorbing host medium Part. Part. Syst. Charact. 13 89-96

[31] Quinten M 2011 Optical Properties of Nanoparticle Systems : Mie and Beyond (New York: Wiley)

[32] Schnaiter M and Mutschke H 1998 Matrix-isolated nano-sized carbon grains as an analog for the 217.5 nanometer feature carrier Astrophys. J. 498 486-96

[33] Polder D and van Santeen J H 1946 The effective permeability of mixtures of solids Physica 12 257-71

[34] Granqvist C G and Hunderi O 1977 Optical properties of ultrafine gold particles Phys. Rev. B 16 3413-534 